\section{Alternatively spliced fibronectin extra domain $A$ is required for hemangiogenic recovery upon bone marrow chemotherapy}

In the bone marrow (BM), the hematopoietic stem cells (HSCs) give rise to all blood cell lineages. ${ }^{1}$ The maintenance, differentiation and proliferation of HSCs are regulated in both cell-autonomous (e.g. GATA-1 or PU.1 transcription factors) and non-cell-autonomous ways. The non-cellautonomous regulation of HSCs requires factors important for the proliferation, mobilization, homing and engraftment that are produced by specialized cells surrounding HSCs. In the BM perivascular niche, both mesenchymal stromal cells (MSCs), such as CXCL12-abundant reticular cells (CAR cells) ${ }^{2}$ and nestin $+\mathrm{MSCs}^{3}{ }^{3}$ arteriolar $\mathrm{NG}^{+}$pericytes, ${ }^{4}$ as well as sinusoidal VEGFR3-endothelial cells, ${ }^{5}$ have all been shown to express pro-hematopoietic paracrine factors during both homeostatic hematopoiesis and regeneration of the hematopoietic system following cytotoxic stress.

Despite advances in the understanding of HSC biology, mechanisms of $\mathrm{BM}$ regeneration after chemotherapy are not completely understood. The extracellular matrix (ECM) of the BM comprises fibrous proteins such as types I and IV collagen and fibronectin (FN), and non-fibrous proteins such as tenascin- $\mathrm{C}$ and proteoglycans. Recently, tenascin- $\mathrm{C}$ was found to be dramatically up-regulated during hematopoietic recovery after myeloablation and to promote HSC proliferation through $\alpha 9 \beta 1$ engagement, ${ }^{6}$ while products of ECM breakdown like hyaluronate were demonstrated to act as inflammatory signals during BM recovery and to affect HSC function and differentiation.? Following insult to the BM microenvironment, cells engage Toll Like Receptor 4 (TLR4) resulting in secretion of proinflammatory cytokines such as TNF $\alpha$ and IL- 6 that acti-
A
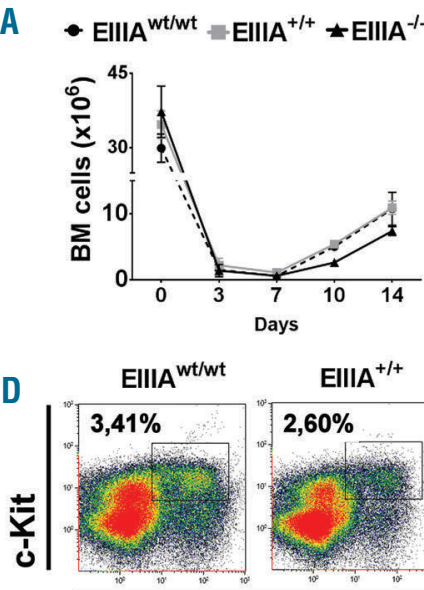

EIIIA ${ }^{+/+}$

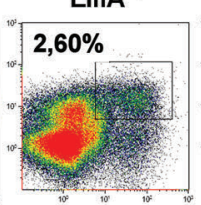

Sca-1

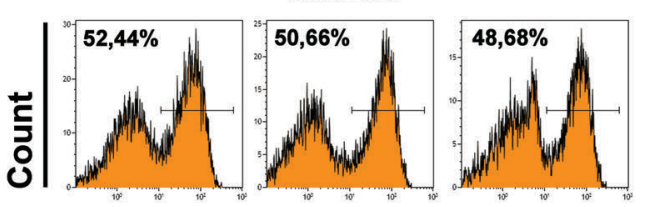

Gate: LSK

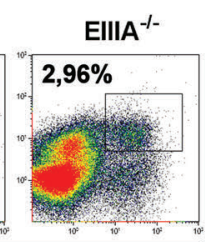

B

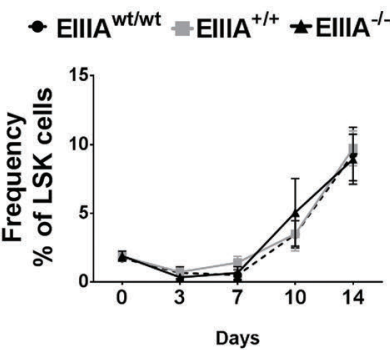

E
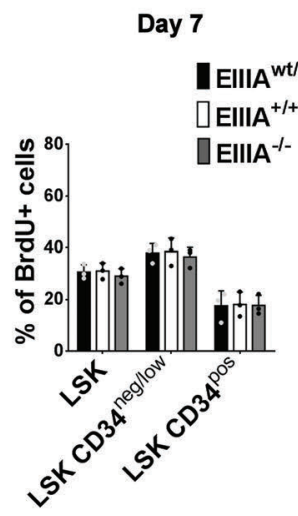

C

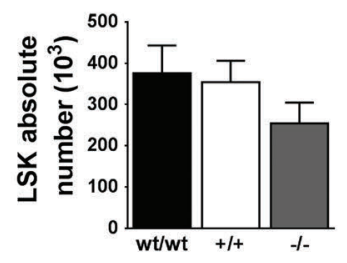

F

Day 10

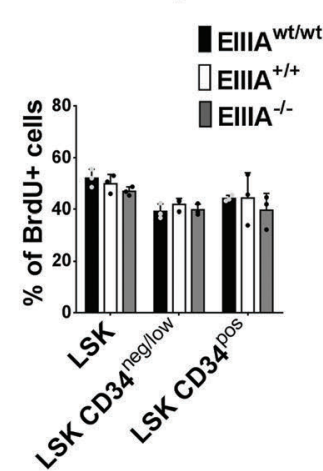

G

\title{
BrdU
}
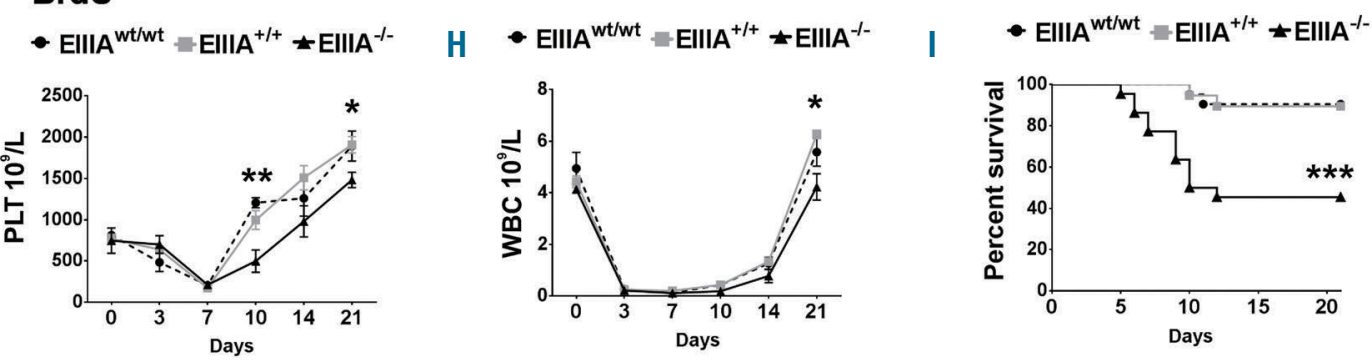

Figure 1. Impaired bone marrow (BM) recovery in mice lacking EIIIA FN after 5-fluorouracil (5-FU) administration. (A) Time course analysis of BM cell count in wild-type (WT), EIIIA ${ }^{++}$and EIIIA $\%$ mice during 5-FU treatment. (B) Time course analysis of LSK (Lineage-/Sca-1 $1^{+} / \mathrm{c}^{-K i t^{+}}$) frequency by FACS after 5-FU treatment. $(n=4)$. (C) Absolute number of LSKs at day 10 of 5-FU treatment. $(n=4)$. (D) Representative flow cytometry analysis of in vivo BrdU incorporation in LSK cells at day 10 of 5-FU treatment. (E) Percentages of BrdU+ cells at day 7 of 5-FU treatment. LSK: Lineage-/Sca-1+/C-Kit'; ST-HSC+MMPs=LSK CD34+; LT-HSC: LSK

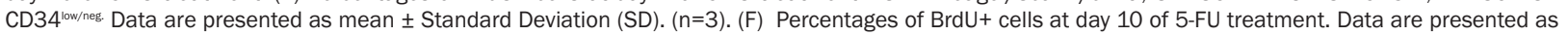
mean $\pm S D$. $(n=3)$. ( $(G$ and H) Time course analysis of peripheral platelet $(G)$ and white $(H)$ blood cell counts during 5 -FU treatment. ( $n=4$ mice in any experimental point for each group). (I) Kaplan-Meier survival curves of WT, ElIIA ${ }^{+/+}$and ElIIA treated with 5-FU; $\mathrm{n}=20,23,20$ respectively. All data are expressed as mean \pm SD. $* P<0.05, * * P<0.01, * * * P<0.001$. 
vate NF- $\alpha \kappa B$ signaling. Several pro-inflammatory cytokines are, therefore, considered new regulators of HSC function. ${ }^{8}$ In this regard, interleukin-1 (IL-1), produced by BM endothelial cells, has been demonstrated to increase HSC differentiation and myelopoiesis.

Prompt deposition of FN-rich extracellular matrix is a critical feature of tissue healing and the host response to injury. The alternatively spliced FN variant containing the extra domain A (FN EIIIA) is expressed at sites of injury, chronic inflammation, and solid tumors. ${ }^{10}$ Although its function is not well understood, FN EIIIA has been previously linked to a variety of in vitro cellular events and in vivo molecular outcomes. Increased expression of EIIIA+ isoform modulates cell cycle progression, myofibroblast differentiation, ${ }^{11}$ prothrombotic effects on platelets, ${ }^{12}$ and protection of vessel walls in conditions of disturbed flow. ${ }^{13}$ These events are mediated by the ability of the EIIIA domain to bind the TLR4 and leukocyte integrins, such as $\alpha 9 \beta 1, \alpha 4 \beta 1$ and $\alpha 4 \beta 7 .{ }^{14,15}$ However, effects of EIIIA FN variant on hematopoietic stem cell behavior remain largely unknown. We recently demonstrated that during physiological hematopoiesis a small fraction of BM-derived FN contains the EIIIA domain and that mice constitutively with (inclusion, EIIIA ${ }^{+/+}$) or without (exclusion, EIIIA ${ }^{-/}$) the EIIIA exon present comparable levels of hematopoietic stem cells, myeloid and lymphoid progenitors in the BM. ${ }^{16}$ Moreover, only minor alterations were detected in blood parameters and in hematopoietic frequencies of BM granulocytes/monocytes and B cells. Thus, given the role of EIIIA exon splicing during healing processes, in the present work we studied the role of EIIIA FN isoform in physiological $\mathrm{BM}$ regeneration, taking advantage of mouse strains unable to undergo a regular alternative splicing of the EIIIA exon.

To investigate the role of EIIIA domain of FN in BM regeneration, we analyzed $\mathrm{BM}$ recovery after chemotherapy in mice with aberrant EIIIA exon splicing. Both EIIIA ${ }^{+/+}$ and EIIIA ${ }^{-/}$mice showed comparable BM cell counts and recovery of HSCs (Lineage-/Sca-1+/c-Kit ${ }^{+}, \mathrm{LSK}$ ) up to three weeks of 5-FU treatment with respect to wild-type (WT) controls (EIIIA ${ }^{\mathrm{wt} / \mathrm{wt}}$ ) (Figure 1A-C). A similar proliferation rate was observed by flow cytometry in HSC subsets after $\mathrm{BrdU}$ incorporation at days 7 and 10 of chemotherapy (Figure 1D-F). Similarly, proliferation rates of BM cells were comparable among mice genotypes as evaluated by western blot analysis of the proliferation marker PCNA (Online Supplementary Figure S1A-C). However, immune-phenotyping of BM cells revealed a reduced frequency of megakaryocytes and myeloid cells at days 10 and 14 in the EIIIA ${ }^{-1-}$ mice (Online Supplementary Figure S2). Consistently, delayed recovery of platelets and white blood cells were detected in the EIIIA ${ }^{-/-}$mice (Figure $1 \mathrm{G}$ and $\mathrm{H}$ and Online Supplementary Figure S3). These events resulted in an increased mortality rate of approximately $50 \%$ in EIIIA ${ }^{-1}$ mice as compared to WT and EIIIA ${ }^{+/+}$mice (Figure 1I). To decipher the role of the EIIIA domain during these processes, we measured the inclusion rate of EIIIA exon in individual BM cell lineages using primers encompassing this exon. Interestingly, higher levels of EIIIA FN expression were
A $\int \mathrm{CD} 45^{+} \mathrm{ICD} / \mathrm{CD} / \mathrm{CD} 31^{+} \quad$ B

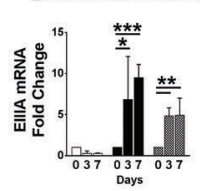

F

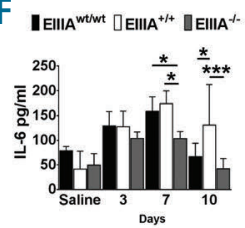

J

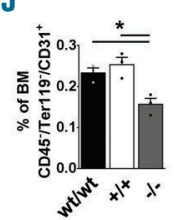

B

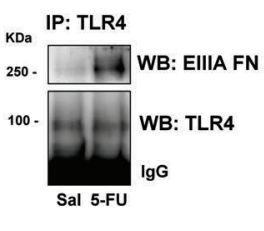

G

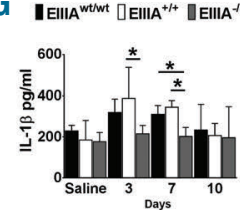

K

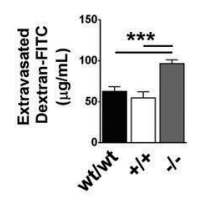

C

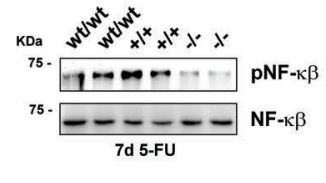

D

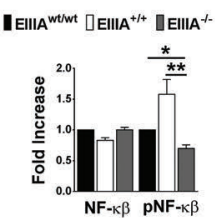

H

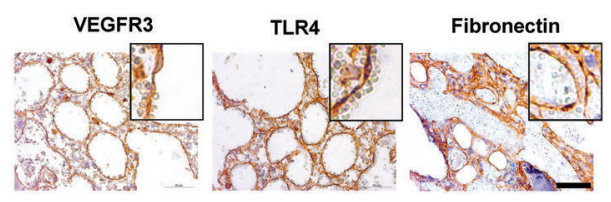

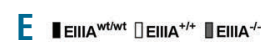

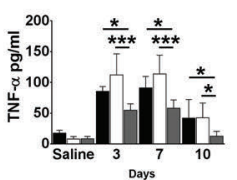

I

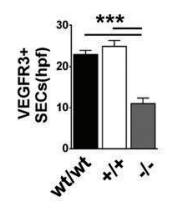

L GATE: CD45-Ter199-/CD 31+

MI GATE: CD45-TTr19--ICD31+

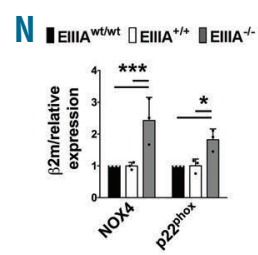

Figure 2. Defective vascular niche re-modeling in the absence of EIIIA FN. (A) RT-PCR of EIIIA FN expression in sorted hematopoietic lineage ${ }^{+}$CD45 $5^{+}$cells, CD 45 /CD31 ${ }^{+}$endothelial cells and CD45 /CD31 /CD140a ${ }^{+}$stromal cells from saline or 5 -fluorouracil (5-FU) wild-type (WT) mice at days 3 and 7 ( $n=3$ ). (B) TLR4 was immunoprecipitated from bone marrow (BM) cells or saline of 3-day old 5-FU treated WT mice and probed with antibodies against the EIIIA domain of FN. (C and D) Western blot analysis of phosphorylated and total NF- $\kappa \beta$ after seven days of 5-FU treatment (C) and relative densitometry analysis (D). (E-G) Amounts of TNF- $\alpha(E)$, IL-6 (F) and IL-1 $\beta(G)$ cytokines in supernatants of BM cells flushed from saline or 5-FU-treated mice ( $n=3$ mice in any experimental point for each group). (H) Immunohistochemistry staining of FN, TLR4 receptor and VEGFR3 on 5-FU-treated BM sections of WT mice at day 7 . Scale bar = 50 $\mu$ m. (I) Quantification of VEGFR3+ vessels on BM sections after seven days of 5-FU treatment $(n=3)$. (J) Frequency of CD45 $/$ Ter119-/CD31 $^{+}$endothelial cells in BM of WT and transgenic mice after seven days of 5-FU treatment $(n=3)$. (K) Quantification of extravasated FITC-Dextran in BM cell-free fluids of WT, EllIA ${ }^{+/+}$and $\mathrm{EIII}^{\%}$ mice at day 7 of 5 -FU treatment $(n=4)$. ( $\mathrm{L}$ and M) Determination of mean fluorescence intensity (MFI) by flow cytometry of the ROS probe CM-H2DCFDA (L) and the nitric oxide (NO) probe DAF-FM (M) in gated CD45-/Ter119-/CD31 endothelial cells at day 6 after 5-FU treatment ( $n=4)$. (N) RT-PCR of NOX4 and p22phox expression relative to $\beta 2$-microglobulin and normalized to WT in sorted CD45/Ter119/CD31 endothelial cells at day 6 after 5 -FU treatment ( $\mathrm{n}=3$ ). All data are expressed as mean \pm Standard Deviation. $* P<0.05, * * P<0.01, * * * P<0.001$. 
detected in enriched $\mathrm{BM}$ populations for endothelial $\left(\mathrm{CD} 45 / \mathrm{CD} 1^{+}\right)$and stromal (CD45/CD31\%CD140a ${ }^{+}$) cells after three and seven days of 5 -FU treatment, while hematopoietic cells $\left(\mathrm{CD} 45^{+}\right)$showed reduced levels of EIIIA FN expression (Figure 2A). Besides sparing HSCs, 5-FUdependent myelosuppression induces the regression of the majority of BM sinusoidal vasculature, inflammation and phagocytic removal of dead cells by macrophages. ${ }^{17}$ Upon binding to TLR4, EIIIA FN activates its signaling pathway in an MyD88-dependent manner, which leads to the activation of NF-кB. ${ }^{14}$ Increased levels of EIIIA/TLR4 interaction were evident in BM cells of WT mice recovered at day 3 of 5-FU treatment (Figure 2B). To demonstrate the interaction of EIIIA FN and TLR4 during the peak of tissue injury, we measured the ability of EIIIA FN to elicit LPS-like responses in injured BM cells through activation of TLR4 downstream signaling pathway. Analysis of NF- $\alpha \kappa \beta$ signaling pathway by western blotting revealed discrepancies in the activation of this pathway, as underlined by the reduced levels of phosphorylated $N F \alpha \kappa \beta$ in BM cells of EIIIA $^{-1}$ mice during 5-FU treatment (Figure 2C and D). Consequently, reduced amounts of pro-inflammatory cytokines (TNF- $\alpha$, IL- 6 and IL1 $\beta$ ) were present in BM supernatants of EIIIA ${ }^{-/}$mice during 5 -FU treatment (Figure $2 \mathrm{E}-\mathrm{G})$.

There is emerging evidence to suggest that most of these pro-inflammatory signals arise from BM endothelium during pan-hematopoietic injury to promote HSC function. ${ }^{18}$ Thus, based on the increased expression of EIIIA FN in BM sinusoidal endothelial cells during chemotherapy, and the positive signals for both TLR4 and FN in regenerating VEGFR $^{+}$endothelial cells of sinusoids after seven days of 5 -FU treatment in WT mice (Figure $2 \mathrm{H}$ ), we focused on the effects of altered EIIIA splicing on 5-FU-dependent vasculature regression.

Lack of EIIIA FN splicing seemed to hamper the endothelial cell maintenance and regeneration required for HSC rebound and mature blood cell release into the bloodstream. Quantification of VEGFR3 ${ }^{+}$sinusoids distribution by immunohistochemistry and CD45/Ter119/CD31+ endothelial cells content by FACS revealed an increased vascular regression in the BM of EIIIA ${ }^{-/}$mice compared to other strains (Figure 2I and J). Consistently, vascular leakage characterized $\mathrm{BM}$ vascular districts of EIIIA ${ }^{-/}$mice after seven days of 5 -FU treatment as determined by the increased retention of injected FITC-Dextran into cell-free BM fluids of these mice (Figure $2 \mathrm{~K}$ ). The biological role of EIIIA FN in endothelial cells is unclear. Recently, lack of EIIIA FN has been demonstrated to worsen endothelial cell function in a mouse model of diabetes by increasing NADPH-oxidase NOX4-derived Reactive Oxygen Species (ROS) production. ${ }^{19}$ Thus, based on this new protective role of EIIIA FN in preventing ROS-dependent endothelial dysfunction, and the reported elevation of ROS production in endothelial cells exposed to 5 -FU, ${ }^{20}$ we measured intracellular ROS production and nitric oxide (NO) levels with fluorescent probes in CD45/Ter119/CD $31^{+}$endothelial cells of mice after 5-FU treatment in vivo. Endothelial cells of EIIIA ${ }^{-1}$ mice were characterized by increased ROS production and decreased NO levels with respect to WT and EIIIA $^{+/+}$mice at day 6 of 5 -FU treatment (Figure 2L and M). Consistently, mRNA levels of NOX4 and of its modulatory subunit p22phox were up-regulated in sorted endothelial cells of EIIIA ${ }^{-/-}$mice with respect to WT and EIIIA $^{+/+}$mice (Figure $2 \mathrm{~N}$ ). In addition, reduced expression of stromal marker $\alpha$-SMA (Online Supplementary Figure S4A and B), as well as lower levels of active MMP-2 and MMP-9 were detected in 5-FU treated EIIIA ${ }^{-/}$mice (Online Supplementary Figure $S 4 C$ and $D$ ) suggesting that EIIIA domain inclusion/exclusion affects stromal remodeling of the vascular compartment after BM injury.

In conclusion, we delineate a new physiological role of EIIIA domain of FN during BM injury. In this scenario, expression of EIIIA FN by endothelial and stromal cells upon BM injury promotes the secretion of a specific milieu of factors supporting BM vascular niche healing and determining a better survival outcome. The absence of EIIIA FN in vivo resulted in abnormal vascular niche healing, as demonstrated by reduced HSC differentiation, stromal activity and increased endothelial oxidative stress. These events were accompanied by a reduction in NF- $\kappa \beta$ signaling and inflammatory cytokines release. Taken together, our findings suggest that alternatively spliced EIIIA FN is required for proper $\mathrm{BM}$ regeneration.

\section{Alessandro Malara, ${ }^{1,2}$ Cristian Gruppi, ${ }^{1}$ Giuseppe Celesti, ${ }^{3}$} Vittorio Abbonante, ${ }^{1,2}$ Gianluca Viarengo, ${ }^{4}$ Luigi Laghi, ${ }^{3}$ Luigi De Marco, ${ }^{5,6}$ Andrés F. Muro and Alessandra Balduini, ${ }^{1,2,8}$

${ }^{1}$ Department of Molecular Medicine, University of Pavia, Italy; ${ }^{2}$ Laboratory of Biotechnology, IRCCS San Matteo Foundation, Pavia, Italy; ${ }^{3}$ Laboratory of Molecular Gastroenterology, Humanitas Clinical and Research Center, Rozzano, Milan, Italy; Immunohaematology and Transfusion Service, Apheresis and Cell Therapy Unit, IRCCS San Matteo Foundation, Pavia, Italy; 'Department of Translational Research, National Cancer Center (IRCCS CRO), Aviano, Italy; ${ }^{6}$ Department of Molecular and Experimental Medicine, The Scripps Research Institute, La Jolla, CA, USA; ${ }^{7}$ The International Centre for Genetic Engineering and Biotechnology, Trieste, Italy and ${ }^{8}$ Department of Biomedical Engineering, Tufts University, Medford, MA, USA.

Acknowledgments: we thank Dr. Francesco Moccia (Department of Biology and Biotechnology, University of Pavia, Italy) for kindly providing reagents used in this study.

Funding: this paper was supported by Cariplo Foundation to $A B$ (2010-0807 and 2013.0717), Italian Ministry of University and Research FIRB to AM (RBFR1299KO), Friuli-Venezia Giulia Regional Grant to AFM and LDM (LR 26/2005).

Correspondence: alessandra.balduini@unipv.it doi:10.3324/haematol.2017.173070

Information on authorship, contributions, and financial \& other disclosures was provided by the authors and is available with the online version of this article at www. haematologica.org.

\section{References}

1. Orkin SH, Zon LI. Hematopoiesis: an evolving paradigm for stem cell biology. Cell. 2008;132(4):631-644

2. Sugiyama T, Kohara H, Noda M, Nagasawa T. Maintenance of the hematopoietic stem cell pool by CXCL12-CXCR4 chemokine signaling in bone marrow stromal cell niches. Immunity. 2006;25(6):977988

3. Méndez-Ferrer S, Michurina TV, Ferraro F, et al. Mesenchymal and haematopoietic stem cells form a unique bone marrow niche. Nature. 2010;466(7308):829-834.

4. Kunisaki Y, Bruns I, Scheiermann C, et al. Arteriolar niches maintain haematopoietic stem cell quiescence. Nature. 2013;502(7473):637643

5. Kopp HG, Hooper AT, Avecilla ST, Rafii S. Functional heterogeneity of the bone marrow vascular niche. Ann N Y Acad Sci. 2009;1176:4754.

6. Nakamura-Ishizu A, Okuno Y, Omatsu Y, et al. Extracellular matrix protein tenascin- $\mathrm{C}$ is required in the bone marrow microenvironment primed for hematopoietic regeneration. Blood 2012;119(23):5429-5437.

7. Matrosova VY, Orlovskaya IA, Serobyan N, Khaldoyanidi SK Hyaluronic acid facilitates the recovery of hematopoiesis following 5-fluorouracil administration. Stem Cells. 2004;22(4):544-555.

8. King KY, Goodell MA. Inflammatory modulation of HSCs: viewing the HSC as a foundation for the immune response. Nat Rev Immunol. 2011;11(10):685-692. 
9. Pietras EM, Mirantes-Barbeito C, Fong S, et al. Chronic interleukin-1 exposure drives haematopoietic stem cells towards precocious myeloid differentiation at the expense of self-renewal. Nat Cell Biol. 2016;18(6):607-618.

10. To WS, Midwood KS. Plasma and cellular fibronectin: distinct and independent functions during tissue repair. Fibrogenesis Tissue Repair. 2011;4:21.

11. Serini G, Bochaton-Piallat ML, Ropraz P, et al. The fibronectin domain ED-A is crucial for myofibroblastic phenotype induction by transforming growth factor-beta1. J Cell Biol. 1998;142(3):873-881.

12. Chauhan AK, Kisucka J, Cozzi MR, et al. Prothrombotic effects of fibronectin isoforms containing the EDA domain. Arterioscler Thromb Vasc Biol. 2008;28(2):296-301.

13. Murphy PA, Hynes RO. Alternative splicing of endothelial fibronectin is induced by disturbed hemodynamics and protects against hemorrhage of the vessel wall. Arterioscler Thromb Vasc Biol. 2014;34(9):2042-2050

14. Okamura $Y$, Watari $M$, Jerud ES, et al. The extra domain A of fibronectin activates Toll-like receptor 4. J Biol Chem. 2001;276(13):10229-10233.

15. Shinde AV, Bystroff C, Wang C, et al. Identification of the peptide sequences within the EIIIA (EDA) segment of fibronectin that mediate integrin alpha9beta1-dependent cellular activities. J Biol Chem. 2008;283(5):2858-2870

16. Malara A, Gruppi C, Celesti G, et al. Brief Report: Alternative Splicing of Extra Domain A (EIIIA) of Fibronectin Plays a TissueSpecific Role in Hematopoietic Homeostasis. Stem Cells. 2016;34(8):2263-2268.

17. Radley JM, Scurfield G. Effects of 5-fluorouracil on mouse bone marrow. Br J Haematol. 1979;43(3):341-351.

18. Mirantes C, Passegué E, Pietras EM. Pro-inflammatory cytokines: emerging players regulating HSC function in normal and diseased hematopoiesis. Exp Cell Res. 2014;329(2):248-254.

19. Gortan Cappellari G, Barazzoni R, Cattin L, Muro AF, Zanetti M. Lack of Fibronectin Extra Domain A Alternative Splicing Exacerbates Endothelial Dysfunction in Diabetes. Sci Rep. 2016;6:37965.

20. Focaccetti C, Bruno A, Magnani E, et al. Effects of 5-fluorouracil on morphology, cell cycle, proliferation, apoptosis, autophagy and ROS production in endothelial cells and cardiomyocytes. PLoS One. 2015;10(2):e0115686. 